\title{
Influence of High-Velocity Blood Flow on Right-to-Left Shunt in Patients with Patent Foramen Ovale during the Valsalva Maneuver
}

\author{
Xiaohui Zhang1,2, Baomin Liu ${ }^{*}$ \\ ${ }^{1}$ Department of Ultrasound, The Second Affiliated Hospital of Xi'an Jiaotong University, Xi'an, China \\ ${ }^{2}$ Department of Ultrasound, Affiliated Hospital of Tibet University for Nationalities, Xianyang, China \\ Email: ${ }^{\star}$ dr.liubm@163.com
}

How to cite this paper: Zhang, X.H. and Liu, B.M. (2019) Influence of High-Velocity Blood Flow on Right-to-Left Shunt in Patients with Patent Foramen Ovale during the Valsalva Maneuver. Journal of Biosciences and Medicines, 7, 13-22.

https://doi.org/10.4236/jbm.2019.71002

Received: November 29, 2018

Accepted: December 30, 2018

Published: January 3, 2019

Copyright $\odot 2019$ by author(s) and Scientific Research Publishing Inc. This work is licensed under the Creative Commons Attribution-NonCommercial International License (CC BY-NC 4.0). http://creativecommons.org/licenses/by-nc/4.0/

\section{Abstract}

In this study, we investigated the changes in the right-to-left shunt (RLS) of the patent foramen ovale (PFO) at different phases of the Valsalva maneuver and analyzed the possible mechanisms. The study population consisted of 57 patients with symptoms highly suggestive of a PFO. These patients had been diagnosed with apsychia, migraine with aura, cerebral infarction, transient ischemic attack (TIA), and cerebral ischemia with unknown cause. Routine echocardiography was performed in all patients to rule out a cardiac malformation. Contrast-transcranial Doppler (c-TCD) and contrast-enhanced transthoracic echocardiography (c-TTE) were used to visualize and quantify the RLS. The standard apical four chamber view was used to observe the changes of $\mathrm{E}$ peak, A peak, and velocity-time integral (VTI) ratio of tricuspid blood flow during the strain phase and release phase of the Valsalva maneuver. Paired t-test was used to compare E peak, A peak, and VTI ratio of tricuspid blood flow during the different phases. The right-to-left shunt across the PFO (PFO-RLS) was graded in the two phases and compared by Kruskal-Wallis test. Compared with the strain phase of the Valsalva maneuver, the parameters of E, A, and VTI in diastolic period in patients with PFO-RLS at the release phase were significantly increased $[54.30 \pm 13.65 \mathrm{~cm} / \mathrm{s}$ vs $100.35 \pm 21.11$ $\mathrm{cm} / \mathrm{s}, 42.21 \pm 12.32 \mathrm{~cm} / \mathrm{s}$ vs $57.30 \pm 18.88 \mathrm{~cm} / \mathrm{s}, 10.34 \pm 3.27 \mathrm{~cm} / \mathrm{s}$ vs $19.58 \pm$ $4.56 \mathrm{~cm} / \mathrm{s}$, respectively], and the difference was statistically significant. The positive consequence of PFO-RLS, as diagnosed by c-TTE with the Valsalva maneuver at the release phase of the Valsalva maneuver, was significantly higher than that at the strain phase of the Valsalva maneuver. At the beginning of release phase of the Valsalva maneuver, decreased intrathoracic pressure led to increased venous backflow into the right atrium. Thus, high-velocity blood flow rapidly pushed the PFO open, which resulted in a 
significant increase in the PFO-RLS. Therefore, the increase of the PFO-RLS during the Valsalva maneuver is caused by the impact of high-velocity blood flow the PFO.

\section{Keywords}

Patent Foramen Ovale, Right to Left Shunt, Valsalva Maneuver, High-Velocity Blood Flow

\section{Introduction}

A patent foramen ovale ( $\mathrm{PFO}$ ) is a unique form of atrial septal communication and a congenital heart disease. It is a stealth shunt caused by incomplete fusion of the primum atrial septum and the secundum atrial septum after birth. Previous studies have shown that about $27 \%$ of the general population has a PFO [1] [2]. A PFO is related to many clinical diseases, such as transient ischemic attack, migraine, cerebral infarction, and apsychia with unknown cause [3] [4] [5] [6]. The existence of the right-to-left shunt (RLS) and shunt volume across the PFO during the resting state and under the stress state have become important issues in clinical research in recent times [7]. Several studies have shown that contrast-enhanced transthoracic echocardiography (c-TTE) with Valsalva maneuver can effectively detect the presence of an RLS and determine the shunt volume in patients with PFO in the resting and stress states [8] [9]. Studies have investigated the RLS with the help of a Valsalva maneuver and attributed it to an elevation in right atrial pressure [10]. However, we have observed in clinical practice that the RLS is not obvious when the right atrial pressure is significantly elevated at the strain phase of the Valsalva maneuver, while the RLS increased when the right atrial pressure decreased in the release phase. Therefore, we have a different theory regarding the PFO-RLS mechanism, and this study aimed to provide an alternative explanation for the PFO-RLS, as seen during the Valsalva maneuver.

\section{Materials and Methods}

\subsection{Patient Population}

We performed a prospective study that included 57 patients $(26$ men and 31 women, mean age $39 \pm 13$ [16 - 65] years) with a high suspicion of a PFO. We collected the patents samples from the Second Affiliated Hospital of Medicine School, Xi'an Jiaotong University, during November 2016 to June 2017, and the patents had been diagnosed with conditions such as apsychia, migraine with aura, cerebral infarction, transient ischemic attack and cerebral ischemia with unknown cause. All patients were examined by computed tomography (CT) or magnetic resonance imaging (MRI) to exclude intracranial, extracranial, and pulmonary vascular malformations. Routine transthoracic echocardiography was used to exclude structural cardiac abnormalities. Contrast-transcranial 
Doppler (c-TCD) and contrast-enhanced transthoracic echocardiography (c-TTE) were also used. All patients were taught and made to practice the Valsalva maneuver. Patients were also informed of the patients of the examination methods and procedures. Patients with an acute infection, serious cardiac and renal insufficiency, atrial fibrillation, serious heart valve disease, blood hypercoagulable state, superior vena cava and right atrial thrombosis, or cognitive dysfunction, and those who were unable to perform the Valsalva maneuver during c-TTE, were excluded. All the patients or their relatives had previously given signed informed consent. The study protocol was performed with approval by the ethics committee of the Second Affiliated Hospital of Xi'an Jiaotong University and was performed in accordance with the CONSORT 2010 guidelines.

\section{2. c-TTE Imaging}

The study used the color Doppler ultrasound diagnostic apparatus, the Netherlands Philips IE33 (Philips Healthcare, DA Best, The Netherlands) with S5-1 adult heart probe (frequency: 2 - $5 \mathrm{MHz}$ ). A pressure-measuring device made from a table sphygmomanometer was used. The cuff of the table sphygmomanometer was removed; the finer end of a disposable plastic hose was connected to the rubber tube of the sphygmomanometer, and the wider end was used as the suction end. The device was used to monitor the intrathoracic pressure when patients performed the Valsalva maneuver and were blowing into the plastic hose.

\subsection{Preparation of Agitated Saline}

A $10 \mathrm{ml}$ syringe was used to extract $8 \mathrm{ml}$ normal saline, while another $10 \mathrm{ml}$ syringe was used to extract $1 \mathrm{ml}$ of air. The two syringes were connected to three-limb tubes while extracting $1 \mathrm{ml}$ of blood from the patient. The valve direction of the three-limb tubes was adjusted. Saline and air were mixed in two syringes, and the syringes were pushed back and forth at least 20 times to produce a fine and evenly mixed liquid filled with microbubbles [10].

\subsection{Examination Methods}

Patients were asked to lie in the left recumbent position at a 45 degree angle. They were examined with routine echocardiography and electrocardiography. The apical four-chamber view was recorded. When the heart rate of the patients stabilized, the sampling container was placed on the right ventricular side of the tricuspid valve. The pulse Doppler was used to observe the blood flow spectrum at the tricuspid valve during the resting state, the strain phase and the release phase of the Valsalva maneuver. The tricuspid E peak value, A peak value, and velocity-time integral (VTI) were measured three times in the diastolic period, and the results were averaged. To monitor the strain phase and the release phase of the Valsalva maneuver, patients were asked to remain in the left recumbent position at 45 degrees, and were made to hold the plastic hose of the pressure-measuring device, while sucking on the wider end of the hose. The patients 
were instructed to perform the suction maneuver without allowing for any leakage. Patients performed the standard Valsalva maneuver and blew vigorously until a pressure of $40 \mathrm{mmHg}(1 \mathrm{mmHg}=0.133 \mathrm{kPa})$ was reached, while holding their breath for about 15 seconds [11]. During this period, the blood flow spectrum image at the tricuspid valve in the strain phase of the Valsalva maneuver was recorded. Patients were kept in the original position and were asked to blow out air rapidly, while the tricuspid valve blood flow spectrum was recorded. All patients performed the Valsalva maneuver at least twice. After every measurement, patients were asked to remain in the same position and rest for 5 minutes. This was repeated until the cardiac function returned to the initial state.

\section{5. c-TTE Method and PFO-RLS Grading}

Patients were asked to assume the left recumbent position at a 45 degree angle, and were examined with routine echocardiography. The apical four-chamber view was taken. When the heart rate of patients had stabilized, agitated saline was pushed through the established intravenous pathway in the left upper limb in a bolus. Microbubbles in the left atrium and left ventricle were observed. This examination was performed during the resting state and during the standard Valsalva maneuver and was repeated once or twice. RLS grading was based on the number of microbubbles in the left atrium and left ventricle observed in a single frame image. Level 0 -there is no microbubble in the left atrium and left ventricle, represented as "-"; level 1 (small RLS)-1 to 10 microbubbles per frame image in the left atrium and left ventricle, represented as "+"; level 2 (medium RLS) - 11 to 30 microbubbles per frame image in the left atrium and left ventricle, represented as "++"; level 3 (large RLS)-more than 30 microbubbles per frame image in the left atrium and left ventricle or the left atrium and left ventricle are almost filled with microbubbles, resulting in a significant reduction in atrial and ventricular transparency, represented as “+++" [9].

\subsection{Statistical Analysis}

A statistical analysis was conducted with the software SPSS 21.0. The tricuspid blood flow spectrum parameters E, A, and VTI showed normal distribution. Measurement data was shown as mean \pm standard deviation $(\bar{x} \pm s)$. Paired $\mathrm{t}$-test was used to compare the E, A, and VTI of the blood flow spectrum during the resting state, the strain phase, and the release phase of the Valsalva maneuver. The measurement data was depicted in percentage. Kruskal-Wallis test was used to compare the PFO-RLS grading in the strain phase and the release phase. A $p$ value less than 0.05 was considered statistically significant.

\section{Results}

\subsection{The Socio-Demographic and Clinical Characteristics of the Patients}

AS shown in Table 1, we performed this study that included 57 patients (26 men 
and 31 women, mean age $39 \pm 13$ [16 - 65] years) with a high suspicion of a PFO. And the patents had been diagnosed with conditions such as apsychia, migraine with aura, cerebral infarction, transient ischemic attack and cerebral ischemia with unknown cause.

\subsection{PFO-RLS at Different Phases of the Valsalva Maneuver}

As seen in Table 2, a large PFO-RLS was detected at the release phase of the Valsalva maneuver, in 43 out of 57 cases (75.4\%). However, at the strain phase a large PFO-RLS was detected in 11 out of 57 cases $(19.3 \%)$, there was none or only a small RLS at the strain phase, seen in 46 cases $(80.7 \%)$. This indicates that a large PFO-RLS was detected at the release phase of the Valsalva maneuver, while the PFO-RLS was not obvious during the strain phase. The positive consequence of PFO-RLS was significantly increased at the release phase of the Valsalva maneuver (Figure 1).

Table 1. The socio-demographic and clinical characteristics of the patients and PFO-RLS grading, tricuspid blood flow spectrum parameters at during different phases of Valsalva maneuver.

\begin{tabular}{|c|c|c|}
\hline Variable & Cases $(\mathrm{n}=57)$ & \\
\hline \multicolumn{3}{|l|}{ Age (y) } \\
\hline $16-65$ & 57 & \\
\hline \multicolumn{3}{|l|}{ Sex } \\
\hline Female & $31(54.4 \%)$ & \\
\hline Male & $26(45.6 \%)$ & \\
\hline \multicolumn{3}{|l|}{ Medical history } \\
\hline apsychia & $24(42.1 \%)$ & \\
\hline migraine with aura & $24(42.1 \%)$ & \\
\hline cerebral infarction & $12(21.1 \%)$ & \\
\hline $\begin{array}{l}\text { transient ischemic attack and cerebral } \\
\text { ischemia with unknown cause }\end{array}$ & $35(61.4 \%)$ & \\
\hline PFO-RLS grading & $\begin{array}{l}\text { the strain phase of the } \\
\text { Valsalva maneuver }\end{array}$ & $\begin{array}{l}\text { the release phase of the } \\
\text { Valsalva maneuver }\end{array}$ \\
\hline 0 & $30(52.6 \%)$ & $9(15.8 \%)$ \\
\hline 1 & $16(28.1 \%)$ & $5(8.8 \%)$ \\
\hline 2 & $7(12.3 \%)$ & $11(19.3 \%)$ \\
\hline 3 & $4(7.0 \%)$ & $32(56.1 \%)$ \\
\hline \multicolumn{3}{|l|}{ Tricuspid blood flow spectrum parameters } \\
\hline $\mathrm{E}(\mathrm{cm} / \mathrm{s})$ & $54.30 \pm 13.65$ & $100.35 \pm 21.11$ \\
\hline $\mathrm{A}(\mathrm{cm} / \mathrm{s})$ & $42.21 \pm 12.32$ & $57.30 \pm 18.88$ \\
\hline VTI $(\mathrm{cm} / \mathrm{s})$ & $10.34 \pm 3.27$ & $19.58 \pm 4.56$ \\
\hline
\end{tabular}


Table 2. PFO-RLS during Valsalva maneuver achieved by c-TTE [case(\%)].

\begin{tabular}{cccccc}
\hline Time & Case & $\begin{array}{c}\text { Level 0 } \\
(-)\end{array}$ & $\begin{array}{c}\text { Level 1 } \\
(+)\end{array}$ & $\begin{array}{c}\text { Level 2 } \\
(++)\end{array}$ & $\begin{array}{c}\text { Level 3 } \\
(+++)\end{array}$ \\
\hline The strain phase & 57 & $30(52.63 \%)$ & $16(28.07 \%)$ & $7(12.28 \%)$ & $4(7.02 \%)$ \\
The release phase & 57 & $9(15.79 \%)$ & $5(8.77 \%)$ & $11(19.30 \%)$ & $32(56.14 \%)$ \\
\hline
\end{tabular}
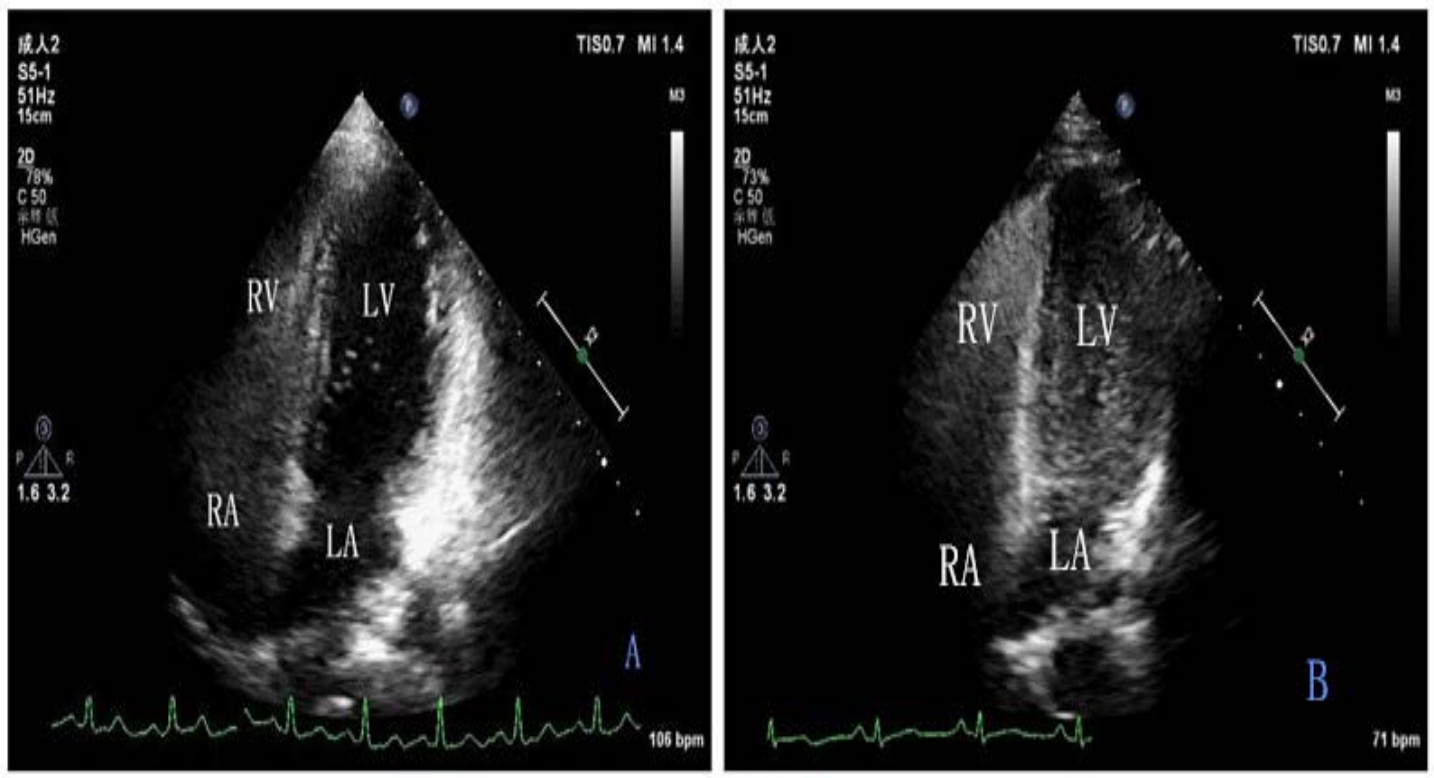

Figure 1. TTE with bubble study during the strain phase (A) and the release phase of the Valsalva maneuver (B).

\subsection{Changes in Tricuspid Blood Flow during the Different Phases of the Valsalva Maneuver}

Compared to the resting state of the Valsalva maneuve, E, A, and VTI on the tricuspid blood flow spectrum decreased at the strain phase of the Valsalva maneuve. However, compared with the strain phase of the Valsalva maneuve, $\mathrm{E}$ value, A value, and VTI on the tricuspid blood flow spectrum increased at the release phase of the Valsalva maneuve, and the observed high-velocity blood flow was almost double of what was observed during the resting state (Table 3 , Figure 2). The difference was statistically significant $(P<0.05)$. This indicates that the tricuspid blood flow accelerated during release phase of the Valsalva maneuve, then in the release phase of the Valsalva maneuve a large PFO-RLS was detected.

\section{Discussion}

Clinical intervention in PFO is dependent on the presence of PFO-RLS and on the shunt volume. Echocardiography is a commonly used technique; however, routine color Doppler flow imaging is only sensitive for left-to-right shunts with relatively high velocity, while a PFO-RLS with relatively low velocity is detected with difficulty. At present, c-TTE is often used to detect and grade PFO-RLS [12]. 
Table 3. Comparison of tricuspid blood flow spectrum parameters during the resting state and at different phases of Valsalva maneuver $(\bar{x} \pm s)$.

\begin{tabular}{ccccc}
\hline Time & Case & $\mathrm{E}(\mathrm{cm} / \mathrm{s})$ & $\mathrm{A}(\mathrm{cm} / \mathrm{s})$ & VTI $(\mathrm{cm} / \mathrm{s})$ \\
\hline The resting state & 57 & $61.14 \pm 12.82$ & $41.89 \pm 10.41$ & $11.74 \pm 3.06$ \\
The strain phase & 57 & $54.30 \pm 13.65$ & $42.21 \pm 12.32$ & $10.34 \pm 3.27$ \\
The release phase & 57 & $100.35 \pm 21.11$ & $57.30 \pm 18.88$ & $19.58 \pm 4.56$ \\
\hline
\end{tabular}

Note: E represents the blood flow velocity of E peak value. A represents the blood flow velocity of A peak value. VTI represents velocity-time integral.

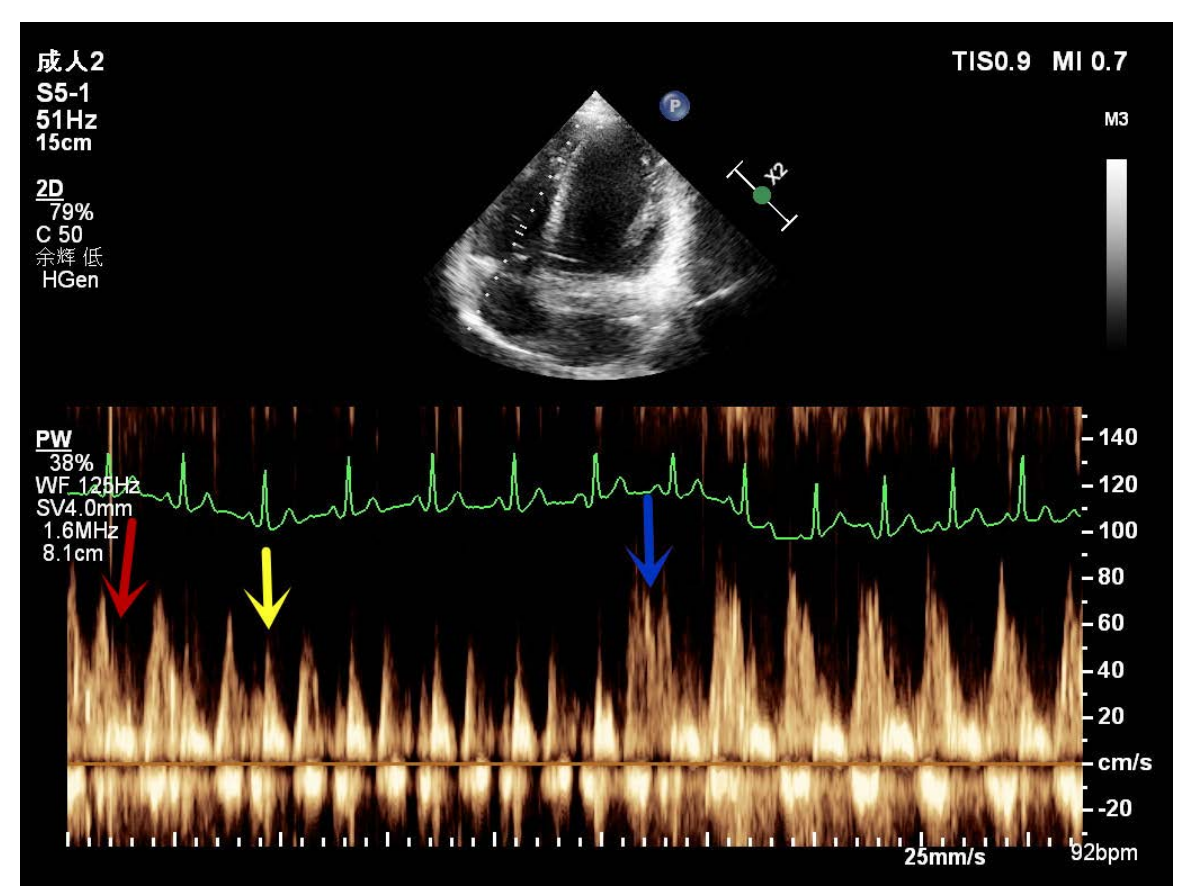

Figure 2. Tricuspid blood flow spectrum at resting state, the strain phase, and the release phase. Resting state indicated the interval time between red arrow and yellow arrow; strain phase referred to interval time between yellow arrow and blue arrow, and blue arrow afterward demonstrated the release phase of Valsalva maneuver.

c-TTE with Valsalva maneuver can significantly increase the detection rate of PFO-RLS [7] [8] [11].

This study showed that the PFO-RLS was not obvious during the strain phase of the Valsalva maneuver, while the positive consequence of PFO-RLS was significantly increased at the release phase. Previous studies often attributed this to the pressure difference between the two atria. A large volume of blood flows back to the right atrium in the release phase of the Valsalva maneuver, raising the pressure in the right atrium relative to the left atrium, which causes the foramen ovale to open and the RLS to increase in magnitude [12] [13] [14].

We did not notice RLS in the strain phase of the Valsalva maneuver when the right atrial pressure and intrathoracic pressure reached a balance of about 40 $\mathrm{mmHg}$; however, RLS was significantly increased at the release phase when the intrathoracic pressure and the right atrial pressure decreased with a quick reflux 
in the superior and inferior vena cave. This phenomenon seems to suggest that the increase in RLS in the release phase of the Valsalva maneuver is mainly due to the impact of the high-velocity blood flow in the right atrium.

Our explanation is as follows: at the beginning of the Valsalva maneuver, the intrathoracic pressure quickly rises to $40 \mathrm{mmHg}$. According to Pascal's law of fluid pressure transfer, walls of both atria, in response to the increase in pressure, transmit the increased pressure to the connected blood vessels. Therefore, the superior and inferior vena cava reflux in the right atrium and the pulmonary vein reflux in the left atrium are blocked. The intraatrial pressure and the bilateral intrathoracic pressure form a balance at $40 \mathrm{mmHg}$, keeping the foramen ovale in its original state. As the Valsalva maneuver continues, reduced filling causes a decrease in the right cardiac blood volume, as well as through the lung tissue and into the left heart, resulting in a decrease in the left cardiac blood volume. Therefore, at the strain phase of the Valsalva maneuver, bilateral atrial pressures increase while the blood flow volume decreases, which causes a "high pressure and low volume" state in both atrial walls. At this time, the atrial pressure difference between the left and right sides become relatively small, such that the PFO-RLS across the foramen ovale becomes undetectable [12] [15].

Upon completion of the Valsalva maneuver, the intrathoracic pressure decreases suddenly, while the systemic circulation receives more blood volume due to the instantaneous return of blood flow from the superior and inferior vena cave to the right atrium, resulting in high-velocity blood flow. This study showed that the VTI of the tricuspid blood flow was $19.58 \mathrm{~cm} / \mathrm{s}$ in the release phase, compared to $10.34 \mathrm{~cm} / \mathrm{s}$ in the strain phase, and the observed high-velocity blood flow was almost double of what was observed during the resting state. Such high-velocity blood flow quickly opens the foramen oval valve, which increases the PFO-RLS magnitude rapidly. This phenomenon is usually significant in the first five cycles of the rapid release phase of the Valsalva maneuver [14] [16]. After the five cardiac cycles, the high-velocity blood flow gradually decreases to normal levels, and the PFO-RLS is significantly reduced.

Therefore, we think that the PFO-RLS increase in the release phase of the Valsalva maneuver is mainly due to the impact of high-velocity blood flow in the right atrium, rather than due to the increase in right atrial pressure, as traditionally believed.

\section{Conclusion}

In conclusion, as the positive intrathoracic pressure increases and the Valsalva maneuver continues in the strain phase, the left and right heart chambers exchange blood through the pulmonary circulatory system; this reduces the pressure difference between the left and right atria, such that the right atrial pressure is slightly higher than or equal to the left atrial pressure, rendering the PFO-RLS undetectable. The intrathoracic pressure decreases rapidly when the release phase comes to an end, and blood flow into the right atrium increases, resulting 
in the opening of the foramen ovale valves, which in turn causes a significant increase in the magnitude of the PFO-RLS. This study sheds some light on our understanding of the hemodynamic changes in the PFO-RLS at different phases of the Valsalva maneuver. The idea proposed in this study may be verified through examination of pressure changes in the left and right atria, with and without Valsalva maneuver, by cardiac catheterization. However, we cannot do cardiac catheterization due to limited resources. We could not verify the pressure changes in the left and right atria at different phases. In addition we will continue to collect more sample sizes and we look forward to further deepening and improving the follow-up work.

\section{Acknowledgements}

This work was supported by the Natural Science Foundation of Tibet Autonomous Region (No: ZRKX2017000133).

\section{Conflicts of Interest}

The authors declare no conflicts of interest regarding the publication of this paper.

\section{References}

[1] Fisher, D.C., Fisher, E.A., Budd, J.H., Rosen, S.E. and Goldman, M.E. (1995) The Incidence of Patent Foramen Ovale in 1,000 Consecutive Patients. A Contrast Transesophageal Echocardiography Study. Chest, 107, 1504-1509. https://doi.org/10.1378/chest.107.6.1504

[2] Hagen, P.T., Scholz, D.G. and Edwards, W.D. (1984) Incidence and Size of Patent Foramen Ovale during the First 10 Decades of Life: An Autopsy Study of 965 Normal Hearts. Mayo Clinic Proceedings, 59, 17-20. https://doi.org/10.1016/S0025-6196(12)60336-X

[3] Cruz-Gonzalez, I., Solis, J., Kiernan, T.J., Yan, B.P., Lam, Y.Y. and Palacios, I.F. (2009) Clinical Manifestation and Current Management of Patent Foramen Ovale. Expert Review of Cardiovascular Therapy, 7, 1011-1022. https://doi.org/10.1586/erc.09.62

[4] Buchholz, S., Shakil, A., Figtree, G.A., Hansen, P.S. and Bhindi, R. (2012) Diagnosis and Management of Patent Foramen Ovale. Postgraduate Medical Journal, 88, 217-225. https://doi.org/10.1136/postgradmedj-2011-130368

[5] Drighil, A., El Mosalami, H., Elbadaoui, N., Chraibi, S. and Bennis, A. (2007) Patent Foramen Ovale: A New Disease? International Journal of Cardiology, 122, 1-9. https://doi.org/10.1016/j.ijcard.2006.12.028

[6] Ghosh, A.K. and Jain, A. (2015) Diagnosis and Management of Patent Foramen Ovale. British Journal of Hospital Medicine, 76, C98-C102. https://doi.org/10.12968/hmed.2015.76.7.C98

[7] Rodrigues, A.C., Picard, M.H., Carbone, A., et al. (2013) Importance of Adequately Performed Valsalva Maneuver to Detect Patent Foramen Ovale during Transesophageal Echocardiography. Journal of the American Society of Echocardiography. Official Publication of the American Society of Echocardiography, 26, 1337-1343. https://doi.org/10.1016/j.echo.2013.07.016 
[8] Wang, W., Huang, H., Liu, Q., et al. (2017) Clinical Values of Transthoracic Echocardiography Combined with Right Heart Contrast Echocardiography in the Right-to-Left Shunt of Patent Foramen Ovale. Journal of Third Military Medical University, 39, 1648-1653.

[9] Zhao, E., Zhang, Y., Kang, C., et al. (2017) Influence of the Valsalva Maneuver on Cardiac Hemodynamics and Right to Left Shunt in Patients with Patent Foramen Ovale. Scientific Reports, 7, 44280. https://doi.org/10.1038/srep44280

[10] Van, H., Poommipanit, P., Shalaby, M., Gevorgyan, R., Tseng, C.H. and Tobis, J. (2010) Sensitivity of Transcranial Doppler versus Intracardiac Echocardiography in the Detection of Right-to-Left Shunt. JACC Cardiovascular Imaging, 3, 343-348. https://doi.org/10.1016/j.jcmg.2009.12.012

[11] Zhao, E., Wei, Y., Zhang, Y., Zhai, N., Zhao, P. and Liu, B. (2015) A Comparison of Transthroracic Echocardiograpy and Transcranial Doppler with Contrast Agent for Detection of Patent Foramen Ovale with or without the Valsalva Maneuver. Medicine, 94, e1937. https://doi.org/10.1097/MD.0000000000001937

[12] Gentile, M., De Vito, A., Azzini, C., Tamborino, C. and Casetta, I. (2014) Adding Blood to Agitated Saline Significantly Improves Detection of Right-to-Left Shunt by Contrast-Transcranial Color-Coded Duplex Sonography. Ultrasound in Medicine \& Biology, 40, 2637-2641. https://doi.org/10.1016/j.ultrasmedbio.2014.06.017

[13] Yue, L.I., Zhai, Y.N., Wei, L.Q., Li, Z. and Ultrasound, D.O. (2013) Comparison of Transthoracic Contrast Echocardiography and Transesophageal Contrast Echocardiography for Detecting Right to Left Shunt in Patients with Petent Foramen Ovale. Chinese Journal of Medical Ultrasound, 10, 44-48.

[14] Li, Y., Liu, R., Zhai, Y., Li, Z. and Ultrasound, D.O. (2014) Preliminary Analysis of Right Heart Contrast Echocardiography in Healthy Volunteers. Chinese Journal of Medical Ultrasound, 11, 135-141.

[15] Zhang, X.Y., Cao, T.S. and Yuan, L.J. (2013) The Mechanics of Left Ventricular Filling during the Strain Phase of the Valsalva Maneuver in Healthy Subjects. The American Journal of the Medical Sciences, 346, 187-189. https://doi.org/10.1097/MAJ.0b013e31826af7de

[16] Thanigaraj, S., Valika, A., Zajarias, A., Lasala, J.M. and Perez, J.E. (2005) Comparison of Transthoracic versus Transesophageal Echocardiography for Detection of Right-to-Left Atrial Shunting Using Agitated Saline Contrast. The American Journal of Cardiology, 96, 1007-1010. https://doi.org/10.1016/j.amjcard.2005.05.061 